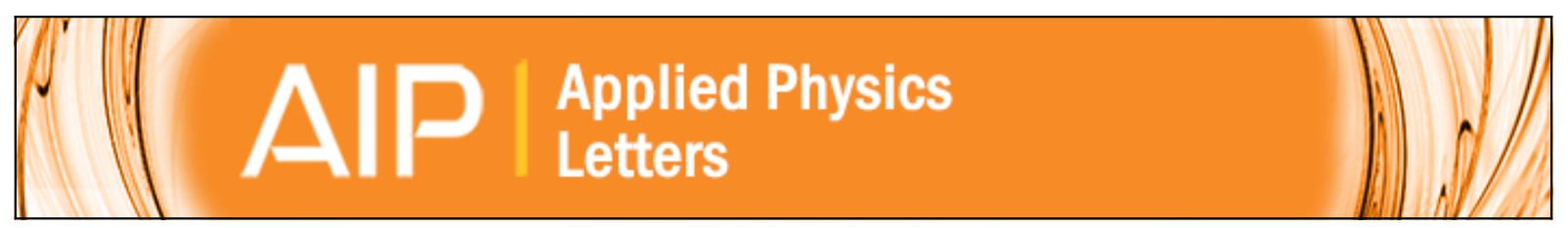

\title{
High-performance silicon nanowire bipolar phototransistors
}

Siew Li Tan, Xingyan Zhao, Kaixiang Chen, Kenneth B. Crozier, and Yaping Dan

Citation: Applied Physics Letters 109, 033505 (2016); doi: 10.1063/1.4959264

View online: http://dx.doi.org/10.1063/1.4959264

View Table of Contents: http://scitation.aip.org/content/aip/journal/apl/109/3?ver=pdfcov

Published by the AIP Publishing

\section{Articles you may be interested in}

High sensitivity silicon single nanowire junctionless phototransistor

Appl. Phys. Lett. 108, 063113 (2016); 10.1063/1.4941807

Evidence of minority carrier injection efficiency $>90 \%$ in an epitaxial graphene/SiC Schottky emitter bipolar junction phototransistor for ultraviolet detection

Appl. Phys. Lett. 108, 043502 (2016); 10.1063/1.4940385

Enhancing the photoresponsivity of bipolar phototransistors for near-infrared detection

Appl. Phys. Lett. 108, 031112 (2016); 10.1063/1.4940395

Silicon-on-insulator based $\mathrm{ZnO}$ nanowire photodetector

J. Vac. Sci. Technol. B 30, 061801 (2012); 10.1116/1.4759261

Low-cost and high-gain silicide Schottky-barrier collector phototransistor integrated on Si waveguide for infrared detection

Appl. Phys. Lett. 93, 071108 (2008); 10.1063/1.2970996

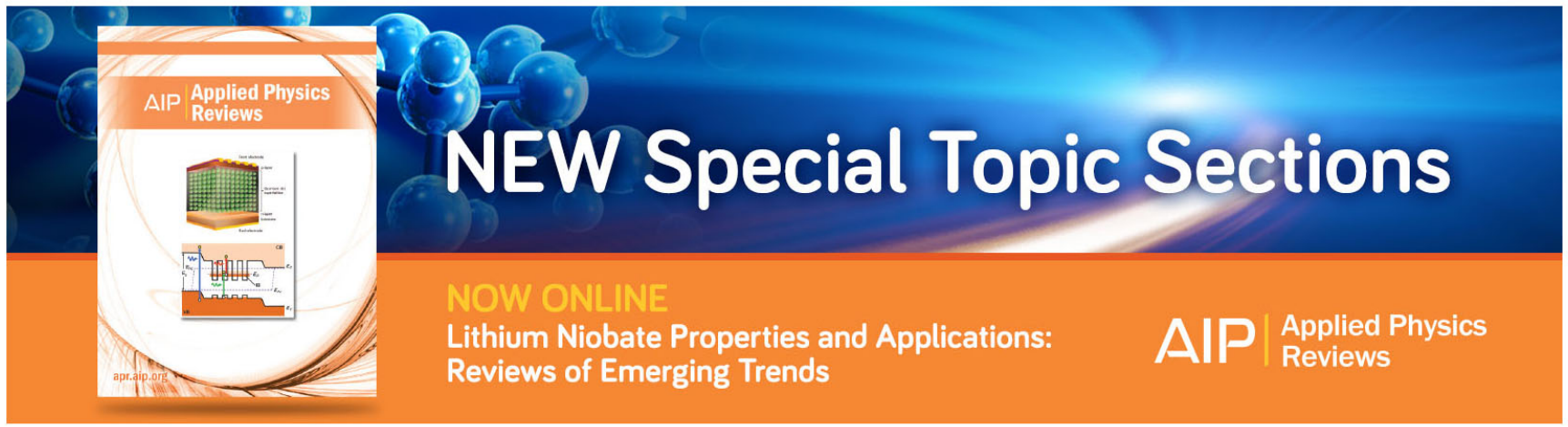




\title{
High-performance silicon nanowire bipolar phototransistors
}

\author{
Siew Li Tan, ${ }^{1, a)}$ Xingyan Zhao, ${ }^{1}$ Kaixiang Chen, ${ }^{1}$ Kenneth B. Crozier, ${ }^{2,3}$ and Yaping Dan ${ }^{1, b)}$ \\ ${ }^{1}$ University of Michigan-Shanghai Jiao Tong University Joint Institute, Shanghai Jiao Tong University, \\ Shanghai 20040, China \\ ${ }^{2}$ Department of Electrical and Electronic Engineering, University of Melbourne, Melbourne, Victoria 3010, Australia \\ ${ }^{3}$ School of Physics, University of Melbourne, Melbourne, Victoria 3010, Australia
}

(Received 23 May 2016; accepted 11 July 2016; published online 20 July 2016)

\begin{abstract}
Silicon nanowires (SiNWs) have emerged as sensitive absorbing materials for photodetection at wavelengths ranging from ultraviolet (UV) to the near infrared. Most of the reports on SiNW photodetectors are based on photoconductor, photodiode, or field-effect transistor device structures. These SiNW devices each have their own advantages and trade-offs in optical gain, response time, operating voltage, and dark current noise. Here, we report on the experimental realization of single SiNW bipolar phototransistors on silicon-on-insulator substrates. Our SiNW devices are based on bipolar transistor structures with an optically injected base region and are fabricated using CMOScompatible processes. The experimentally measured optoelectronic characteristics of the SiNW phototransistors are in good agreement with simulation results. The SiNW phototransistors exhibit significantly enhanced response to UV and visible light, compared with typical Si $p$-i-n photodiodes. The near infrared responsivities of the SiNW phototransistors are comparable to those of $\mathrm{Si}$ avalanche photodiodes but are achieved at much lower operating voltages. Compared with other reported SiNW photodetectors as well as conventional bulk Si photodiodes and phototransistors, the SiNW phototransistors in this work demonstrate the combined advantages of high gain, high photoresponse, low dark current, and low operating voltage. Published by AIP Publishing.

[http://dx.doi.org/10.1063/1.4959264]
\end{abstract}

The miniaturization of semiconductor devices to the subwavelength scale has opened up new possibilities for creating faster, smaller, and more sensitive photodetectors. In recent years, there has been a growing interest in nanowire photodetectors based on $\mathrm{Si}_{1}{ }^{1-9} \mathrm{Ge},{ }^{10,11}$ and various $\mathrm{III}-\mathrm{V}^{12-14}$ and II-VI ${ }^{1,15}$ materials. In the simplest device structure, both ends of a single nanowire are connected to electrodes. High photoconductive gains have been reported in such devices and attributed to the trapping of photogenerated charge carriers by nanowire surface states. ${ }^{4,16}$ On the downside, the performance of nanowire photoconductive devices is typically limited by a slow response time of $>1 \mu \mathrm{s}$ and high dark currents in the nA range. Alternatively, nanowire avalanche photodiodes have shown potential for high optical gain and high-speed response $^{1,2}$ although they typically require a high operating voltage and exhibit excess noise due to the avalanche multiplication process. In bulk devices, bipolar phototransistors (BPT) commonly based on III-V heterojunction materials are capable of providing high optical gain without the drawback of avalanche excess noise. ${ }^{17}$ However, the large base-collector capacitance in conventional bulk bipolar phototransistors imposes a limit on their high-frequency response.

In this letter, we present the experimental demonstration of a single silicon nanowires ( $\mathrm{SiNW}$ ) photodetector based on the bipolar phototransistor structure. Compared with SiNW photodetectors based on photoconductors or $\mathrm{p}-\mathrm{n}$ (or $\mathrm{p}-\mathrm{i}-\mathrm{n}$ )

\footnotetext{
${ }^{a)}$ Currently with the CEA Grenoble, 17 rue des Martyrs, 38054 Grenoble, France.

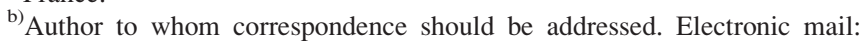
yaping.dan@sjtu.edu.cn
}

junction photodiode structures, ${ }^{1-5}$ our device structure has the simultaneous advantages of high optical gain, low operating voltage, and lower dark current. An obvious strength of nanoscale bipolar phototransistors over their bulk counterparts is that the significantly reduced parasitic capacitance will result in better high-frequency performance. Moreover, the planar SiNW bipolar phototransistor device structure allows for integration with nanoscale antennas to exploit the properties of surface plasmons for photodetection sensitivity enhancement. ${ }^{10,18-20}$

We fabricate single SiNW bipolar phototransistors on a silicon-on-insulator (SOI) wafer in a top-down, CMOScompatible approach (as described in supplementary material Section S1). ${ }^{21}$ Although a higher emitter injection efficiency can be expected in $n p n$-type phototransistors, a pnp-type structure based on a p-type SOI wafer is implemented in this work due to the availability of suitable starting wafers. Fig. 1(a) shows a scanning electron microscopy (SEM) image of a single SiNW phototransistor device after formation of the active regions by ion implantation. The design values of the length, width, and thickness of the SiNW are $3 \mu \mathrm{m}, 200 \mathrm{~nm}$, and $200 \mathrm{~nm}$, respectively. SEM inspection reveals that the actual SiNW length and thickness meet the design values, while the actual width is $\sim 250 \mathrm{~nm}$. Along the length of the nanowire, a pnp device structure is defined with a $1 \mu \mathrm{m} p^{+}$-doped emitter, an $n$-doped base with length ranging from 100 to $500 \mathrm{~nm}$, a collector with $1 \mu \mathrm{m}$ unintentionally doped $\left(p^{-}\right)$section followed by a $p^{+}$-doped sub-collector with length $<1 \mu \mathrm{m}$. Each end of the nanowire is connected to a large-area $p^{+}$-doped contact pad, on which ohmic metal contact is deposited. The desired $p^{+}\left(\sim 5 \times 10^{19} \mathrm{~cm}^{-3}\right)$ and $n\left(\sim 5 \times 10^{17} \mathrm{~cm}^{-3}\right)$ doping levels in the device regions are achieved by boron and 

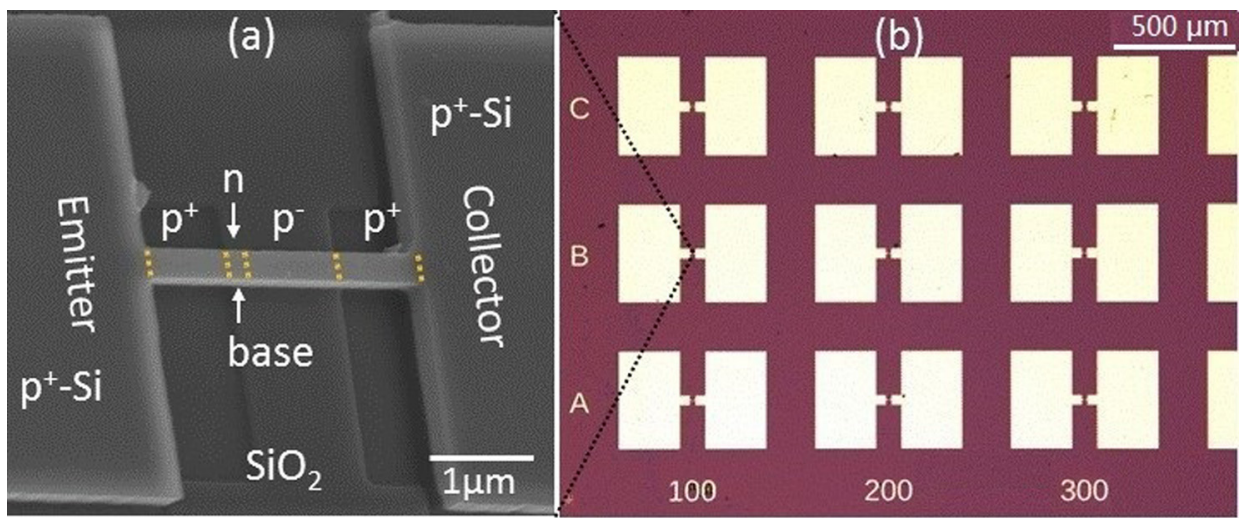

FIG. 1. (a) SEM image of a SiNW pnp phototransistor device structure after ion implantation. The yellow dashed lines are a guide to the eye for the approximate locations of the emitter, base, and collector regions. (b) Optical microscopy image of the device array with metal contacts.

phosphorus ion implantation, respectively, as confirmed by secondary ion mass spectroscopy (SIMS) (see supplementary material, Figure S1.3). ${ }^{21}$ The shallow $\mathrm{SiO}_{2}$ trenches underneath the emitter and sub-collector regions in Fig. 1(a) are imprints caused by the faster etch rate of the boron-implanted $\mathrm{SiO}_{2}$ window regions by buffered oxide etchant (BOE). The optical microscopy image of an array of these devices is shown in Fig. 1(b).

The photocurrent characteristics of the SiNW phototransistors are measured under illumination by a fiber-coupled beam from a diode-pumped solid-state laser (CNI laser MLL$\mathrm{FN}-532-400 \mathrm{~mW}$ ) with a wavelength of $532 \mathrm{~nm}$. The light intensity is varied over four decades by passing the laser beam through neutral density filters. The incident beam intensity is found to be fairly uniform across an area that is over five orders of magnitude larger than the top surface of the SiNW. A commercial photodiode (Hamamatsu G10899-003K) with validated responsivity is used to measure the incident light intensity. Figs. 2(a) and 2(b) compare the measured and simulated photocurrent characteristics. Details regarding these simulations and key parameters are described in supplementary material Section $\mathrm{S} 2$. $^{21}$

It is evident from Fig. 2(a) that the photoresponse of the SiNW phototransistor scales with light intensity over four orders of magnitude, from $3 \mathrm{~mW} / \mathrm{cm}^{2}$ to $30 \mathrm{~W} / \mathrm{cm}^{2}$. At high light intensity $\left(30 \mathrm{~W} / \mathrm{cm}^{2}\right)$, the devices exhibit a high photocurrent density of over $250 \mathrm{~A} / \mathrm{cm}^{2}$ with good reproducibility. In Fig. 2(b), the measured and simulated photocurrent curves at an illumination intensity of $15 \mathrm{~W} / \mathrm{cm}^{2}$ are plotted on a linear scale for a detailed examination. It can be seen from Fig. 2(b) that the SiNW phototransistor device behaves as theoretically predicted and exhibits a weak Early effect at an operating voltage $V_{E} \geq 0.5 \mathrm{~V}$, where a further increase in $V_{E}$ does not result in significant change in the effective base length and photocurrent gain.

The measured dark current level in Fig. 2(a) is below the lower detection limit of our pico-ammeter (100 fA), suggesting that the surface leakage is negligible. For our silicon nanowire devices, this corresponds to a surface recombination velocity (SRV) of $\leq 1000 \mathrm{~cm} / \mathrm{s},{ }^{22,23}$ which is assumed in the simulation. It is also assumed that the electron and hole mobilities in the base and collector regions are an order of magnitude lower than those of intrinsic silicon due to surface roughness scattering ${ }^{12}$ and that the carrier mobilities in the highly $p^{+}$-doped regions are limited by ionized impurity scattering. The carrier mobility values that we assume are within the range of typically low mobility values reported for SiNWs. ${ }^{24,25}$ A comparison between the experimental data (photocurrent vs. light intensity) of a set of SiNW phototransistors with nominal base lengths ranging from 100 to $500 \mathrm{~nm}$ with simulations reveals that the actual base lengths are consistently shorter than their nominal values by approximately $60 \mathrm{~nm}$. For example, Figs. 2(a) and 2(b) show that the simulation results for a device with a base length of $140 \mathrm{~nm}$ are in good agreement with the experimental data for a device with a nominal base length of $200 \mathrm{~nm}$. The narrowing of the base length is possibly caused by diffusion of p-type dopants from the emitter (or other impurities) into the
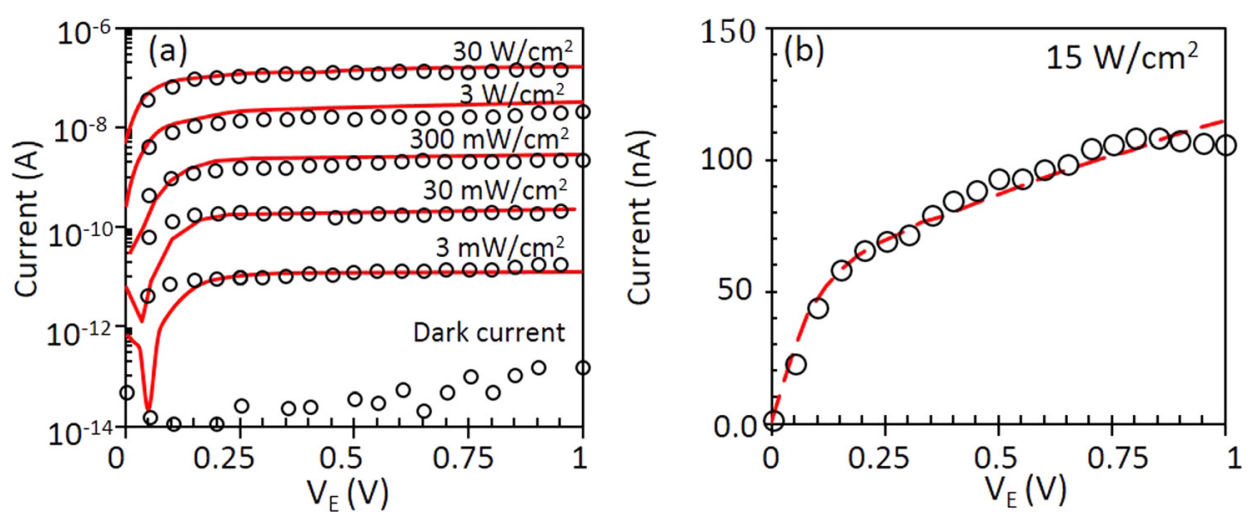

FIG. 2. Measured (open symbols) and simulated (lines) photocurrent characteristics of a SiNW pnp phototransistor with 200-nm nominal base length. (a) Loglinear scale plot of current vs emitter voltage at various illumination intensities (spanning four orders of magnitude) at a wavelength of $\lambda_{0}=532$ nm. (b) Linear-linear scale plot of current vs emitter voltage for an illumination intensity of $15 \mathrm{~W} / \mathrm{cm}^{2}$. The simulated photocurrent curves assume a base length of $140 \mathrm{~nm}$, carrier mobilities equal to $1 / 10$ of bulk values, and a surface recombination velocity (SRV) of $1000 \mathrm{~cm} / \mathrm{s}$. 
base region during the dopant activation by a rapid thermal annealing process.

In Fig. 3(a), we plot photocurrent gain vs nominal base length for a range of illumination intensities. Photocurrent gain is defined as the ratio of the photocurrent of the SiNW phototransistor to the photocurrent of a SiNW $p^{+}-n$ diode (See the inset of Fig. 3(a)) with absorption characteristics equivalent to those of the base-collector junction of the phototransistor, under similar illumination and operating bias conditions. For simplicity, the SiNW diode is chosen to be a $p^{+}-n$ structure with $1.5 \mu \mathrm{m} n$-region rather than a $p^{+}-i-n^{+}$structure with $1 \mu \mathrm{m} i$-region. Optoelectronic simulations predict that the SiNW $p^{+}-n$ and $p^{+}-i-n^{+}$diode structures produce similar levels of primary photocurrent (See the supplementary material, Figure S2.2). ${ }^{21}$ Fig. 3(a) compares the experimental photocurrent gains of a set of SiNW phototransistors with nominal base length ranging from 100 to $500 \mathrm{~nm}$, at different illumination intensities (wavelength $532 \mathrm{~nm}$ ). Fig. 3(a) also contains a plot of simulated photocurrent gain vs effective base length (solid line), which is $60 \mathrm{~nm}$ shorter than the nominal base length (as explained above). The experimental photocurrent gain decreases with increasing base length and begins to saturate at a nominal base length $\geq 300 \mathrm{~nm}$, i.e., at an effective base length $\geq 240 \mathrm{~nm}$. This decrease in gain is known to be the result of the degraded base transport factor in phototransistors with wide base regions. ${ }^{26}$ For a pnp transistor, it means that the fraction of the emitter-injected hole current that reaches the collector is reduced due to recombination with electrons in the base. To minimize the base recombination loss, the nominal base length needs to be $\leq 100 \mathrm{~nm}$.

Although Fig. 3(a) shows that the device with the narrowest base exhibits the highest photocurrent gain of over 200 at low-intensity illumination, the gain decreases gradually with increasing light intensity. This is examined in more detail in Fig. 3(b), which plots photocurrent vs. illumination intensity of the SiNW phototransistors and the equivalent diode. The unity slope of the photocurrent vs illumination intensity plot ( $\log -\log$ scale) indicates that the photocurrent of the SiNW $p^{+}$$n$ diode scales linearly with incident light intensity over a four decade range, which is an expected characteristic of a photodiode. Similarly, the SiNW phototransistors with wider base (nominally $\geq 300 \mathrm{~nm}$ ) also exhibit approximately linear photoresponse with respect to light intensity, albeit with a small gain of $<10$ over the photoresponse of the $p^{+}$- $n$ diode. In contrast, the photocurrent $v s$. light intensity characteristic of the device with 100-nm base becomes sublinear (the slope of the $\log -\log$ plot is less than one) at intensity levels $>100 \mathrm{~mW} / \mathrm{cm}^{2}$. This can be possibly attributed to the charge screening effect of a large number of holes (from $\mathrm{p}+$ emitter) passing across the base-collector depletion region, which narrows the depletion region and extends the effective base length proportionally. The extension of the base length results in the reduction in gain. This gain reduction is negligible for low-gain devices with longer base length ( $\geq 300 \mathrm{~nm}$ ) but becomes relatively significant for those high-gain devices with shorter base lengths at high illumination intensity (Fig. 3(b)).

In the experiments, a few SiNW phototransistor devices on the sample are found to exhibit exceptionally high photocurrent gains (up to $\sim 5500$ ) relative to a SiNW $p^{+}$- $n$ diode, as shown in Figs. 4(a) and 4(b). The high gain characteristics of the device are possibly attributable to the annealing-induced narrowing of the base length to $\leq 40 \mathrm{~nm}$ and strain-induced enhancement of carrier mobilities in the nanowire base and collector regions. ${ }^{27,28}$ The gain characteristics in Fig. 4 have two other distinct features in addition to the exceptionally high values. First, the gain decreases as the light intensity increases (Fig. 4(a)). This is due to the fact that the photocurrent vs light intensity of the nanowire phototransistor shows a sublinear behavior (Fig. 4(b)), resulting in a smaller gain at higher light intensity (larger photocurrent). The sublinearity is possibly caused by the charge screening effect of a large number of holes flowing across the base-collector depletion region, as explained previously. Second, the gain is relatively larger under longer wavelength illumination (Fig. 4(a)), which looks surprising at the first glance. This phenomenon is essentially also related to the photocurrent sublinearity of the device. It is known that silicon is less absorptive at longer wavelength, which results in smaller photocurrent in the device (Fig. 4(b)) although longer wavelength means a higher density of photon flux. A smaller photocurrent at longer wavelength (the same light intensity) leads to a higher gain, according to the photocurrent sublinearity shown in Fig. 4(b).

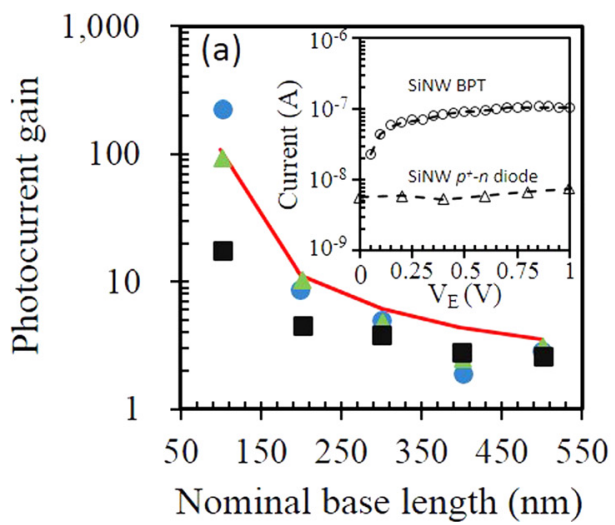

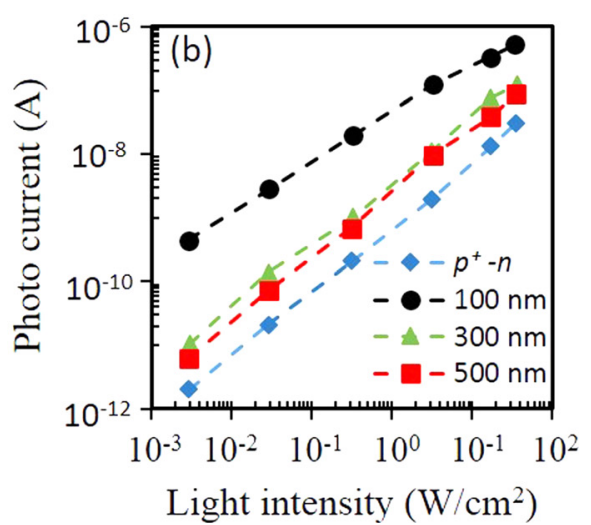

FIG. 3. (a) Measured photocurrent gain values of SiNW pnp phototransistors at $V_{E}=1 \mathrm{~V}$ vs. nominal base length under $3 \mathrm{~mW} / \mathrm{cm}^{2}$ (circles), $300 \mathrm{~mW} / \mathrm{cm}^{2}$ (triangles), and $30 \mathrm{~W} / \mathrm{cm}^{2}$ (squares) illumination intensities (wavelength $\lambda_{0}=532 \mathrm{~nm}$ ). The solid line plots simulated photocurrent gain vs. effective base length $\left(60 \mathrm{~nm}\right.$ shorter than nominal base length) for $300 \mathrm{~mW} / \mathrm{cm}^{2}$ illumination intensity. Inset: Photocurrent of SiNW bipolar phototransistor (BPT) and $p^{+}-n$ photodiode under illumination intensity of $\sim 15 \mathrm{~W} / \mathrm{cm}^{2}$. (b) Measured photocurrent at $V_{E}=1 \mathrm{~V}$ as a function of illumination intensity of SiNW pnp phototransistors with $100-500 \mathrm{~nm}$ nominal base lengths, compared with that of a $p^{+}-n$ diode. The dotted lines are a guide to the eye. 


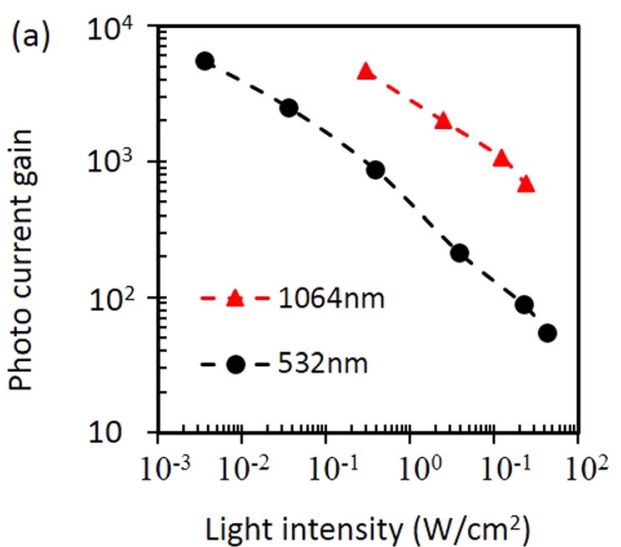

Light intensity $\left(\mathrm{W} / \mathrm{cm}^{2}\right)$

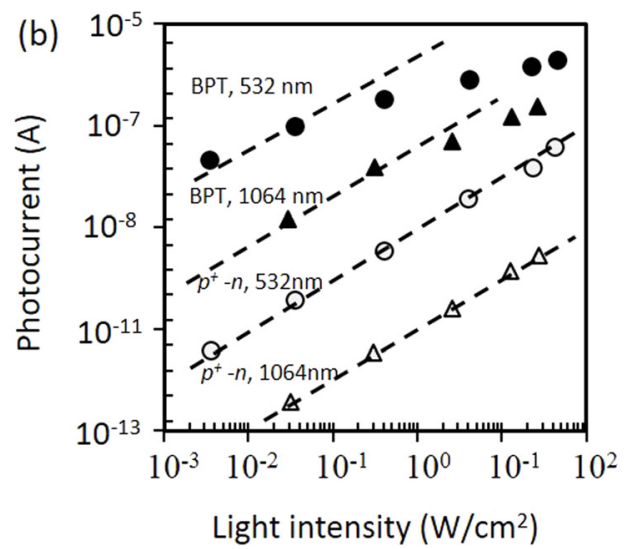

Light intensity $\left(\mathrm{W} / \mathrm{cm}^{2}\right)$
FIG. 4. (a) Measured photocurrent gain characteristics of a SiNW pnp phototransistor device at $V_{E}=1 \mathrm{~V}$ with 100 -nm nominal base length vs illuminations intensities at wavelengths of $532 \mathrm{~nm}$ and $1064 \mathrm{~nm}$. (b) Photocurrent vs illumination intensities (at wavelengths of $532 \mathrm{~nm}$ and $1064 \mathrm{~nm}$ ) of a bipolar phototransistor ("BPT," solid symbols) and a $p^{+}-n$ diode (open symbols). Parallel trend lines (dashed lines) are also shown as guides to the eye (the slope is equal to one).

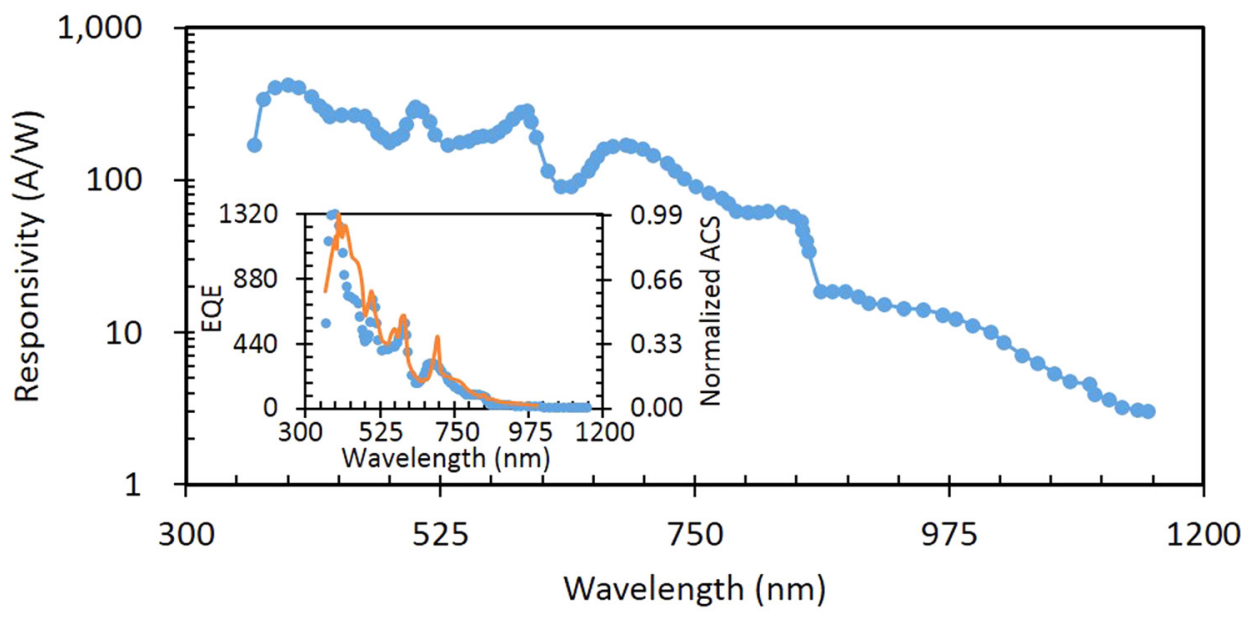

FIG. 5. Spectral responsivity of a highgain SiNW pnp phototransistor device at $V_{E}=1 \mathrm{~V}$ on a log-linear scale. The inset compares the experimental external quantum efficiency (EQE, dots) with simulated absorption crosssection (ACS, solid line) normalized to the peak value, on a linear-linear scale.

The spectral responsivity of the high-gain SiNW phototransistor is measured and plotted as shown in Fig. 5. Measurements are performed with illumination from a grating-based monochromator (HORIBA iHR320) with a focal length of $0.32 \mathrm{~m}$, slit widths of $7 \mathrm{~mm}$, a $250 \mathrm{~W}$ tungsten-halogen white-light source, and the appropriate long-pass filters to remove higher-order wavelengths from the output beam. The output beam of the monochromator is approximately collimated and is coupled into a fiber, whose output illuminates the device. A commercial photodiode (Hamamatsu G10899-003K) with a validated spectral responsivity curve (in $\mathrm{A} / \mathrm{W}$ ) is carefully positioned under illumination conditions similar to those used for the SiNW phototransistor to determine the illumination intensity (in $\mathrm{W} / \mathrm{cm}^{2}$ ). The responsivity at each wavelength is calculated by dividing the measured photocurrent by the optical power incident onto the top surface area of the SiNW (i.e., SiNW surface area multiplied by illumination intensity). The resultant responsivity spectrum has a trend of decrease as the wavelength increases (Fig. 5), due to the fact that the silicon nanowire absorbs less light at longer wavelength. Note that the photo gain is equal to the photoresponsivity of the nanowire phototransistor normalized by that of the $\mathrm{p}^{+}-\mathrm{n}$ nanowire photodiode. As a result, the gain is expected to be a constant independent of wavelength. But it actually increases with the wavelength (Fig. 4(a)) due to the sublinearity effect as previously explained. In the spectrum of Fig. 5, there are ripples in photoresponsivity which are the result of enhanced absorption due to optical resonances of the SiNW, ${ }^{22,28,29}$ as confirmed by simulation (inset in Fig. 5). This leads to a peak responsivity of $\sim 410$ A/W at around $380-390 \mathrm{~nm}$, which is three orders of magnitude higher than the typical ultraviolet (UV) responsivity $(0.2-0.3 \mathrm{~A} / \mathrm{W}$ at $400 \mathrm{~nm})$ of commercial bulk Si photodiodes. ${ }^{30}$ The near-infrared responsivity of $\sim 10 \mathrm{~A} / \mathrm{W}$ at $1000 \mathrm{~nm}$ is comparable to that of a commercial bulk $\mathrm{Si}$ avalanche photodiode $(\mathrm{APD})^{31}$ but is achieved at only a small fraction of the bias voltage of an APD (e.g., $>100 \mathrm{~V}$ for the Si APD of Ref. 31).

In conclusion, the measured optical gain and photoresponse characteristics of our SiNW phototransistors are in good agreement with the predictions of simulations. The devices exhibit good photoresponse linearity over a wide range of illumination intensities. Of noteworthy significance is the ability of the SiNW phototransistor to produce a high gain exceeding 5000, and high responsivity exceeding $100 \mathrm{~A} / \mathrm{W}$ in the $\mathrm{UV}$ and visible ranges, as well as $\sim 10 \mathrm{~A} / \mathrm{W}$ in the near infrared region, at a low operating voltage of $1 \mathrm{~V}$. We believe that the device performance can be enhanced by improving process parameters, in order to achieve higher carrier mobilities and better control of doping profile, especially in the base region. This work has opened up an approach for realizing nanoscale photodetectors, which has shown potential for outperforming conventional photodiodes and phototransistors.

We gratefully acknowledge the ion implantation service performed by Dr. Nianhua Peng at the Surrey Ion Beam Centre, and the use of the e-beam lithography and ICP-RIE facilities at the Center for Nanoscale Systems of Harvard University. 
This work is partly funded by the National "1000 Young Scholars" Program, the National Science Foundation of China (NSFC Grant No. 61376001), Pujiang Talent Program of the Shanghai municipal government, and the China Postdoctoral Science Foundation (Grant No. 2014M551411). K.B.C. acknowledges support from VESKI and the Australian Research Council (FT140100577 and DP150103736). The authors declare no competing financial interest.

${ }^{1}$ O. Hayden, R. Agarwal, and C. M. Lieber, "Nanoscale avalanche photodiodes for highly sensitive and spatially resolved photon detection," Nat. Mater. 5(5), 352-356 (2006).

${ }^{2}$ C. Yang, C. J. Barrelet, F. Capasso, and C. M. Lieber, "Single p-type/intrinsic/n-type silicon nanowires as nanoscale avalanche photodetectors," Nano Lett. 6(12), 2929-2934 (2006).

${ }^{3}$ A. Zhang, S. F. You, C. Soci, Y. S. Liu, D. L. Wang, and Y. H. Lo, "Silicon nanowire detectors showing phototransistive gain," Appl. Phys. Lett. 93(12), 121110 (2008).

${ }^{4}$ A. Zhang, H. Kim, J. Cheng, and Y.-H. Lo, "Ultrahigh responsivity visible and infrared detection using silicon nanowire phototransistors," Nano Lett. 10(6), 2117-2120 (2010).

${ }^{5} \mathrm{M}$. Ziaei-Moayyed and M. Okandan, "CMOS-compatible gate-all-around silicon nanowire detector," in 2011 IEEE Sensors (IEEE, 2011), p. 4.

${ }^{6}$ S. Lee, S. W. Jung, S. Park, J. Ahn, S. J. Hong, H. J. Yoo, M. H. Lee, and D. I. Cho, "Ultra-high responsivity, silicon nanowire photodetectors for retinal prosthesis," in 25th IEEE International Conference on Micro Electro Mechanical Systems (MEMS), Paris, France, 29 January-02 February, 2012 (IEEE, Paris, France, 2012).

${ }^{7}$ H. Park, Y. Dan, Y. J. Yu, P. K. Duane, M. Wober, and K. B. Crozier, "Filter-free image sensor pixels comprising silicon nanowires with selective color absorption," Nano Lett. 14(4), 1804-1809 (2014).

${ }^{8} \mathrm{H}$. Park and K. B. Crozier, "Elliptical silicon nanowire photodetectors for polarization-resolved imaging," Opt. Express 23(6), 7209-7216 (2015).

${ }^{9}$ H. Park and K. B. Crozier, "Vertically stacked photodetector devices containing silicon nanowires with engineered absorption spectra," ACS Photonics 2(4), 544-549 (2015).

${ }^{10}$ L. Tang, S. E. Kocabas, S. Latif, A. K. Okyay, D.-S. Ly-Gagnon, K. C. Saraswat, and D. A. B. Miller, "Nanometre-scale germanium photodetector enhanced by a near-infrared dipole antenna," Nature Photon. 2(4), 226-229 (2008).

${ }^{11}$ A. Solanki and K. Crozier, "Vertical germanium nanowires as spectrallyselective absorbers across the visible-to-infrared," Appl. Phys. Lett. 105(19), 191115 (2014).

${ }^{12}$ A. C. Ford, J. C. Ho, Y.-L. Chueh, Y.-C. Tseng, Z. Fan, J. Guo, J. Bokor, and A. Javey, "Diameter-dependent electron mobility of InAs nanowires," Nano Lett. 9(1), 360-365 (2009).

${ }^{13}$ A. d., L. Bugallo, M. Tchernycheva, G. Jacopin, L. Rigutti, F. H. Julien, S.-T. Chou, Y.-T. Lin, P.-H. Tseng, and L.-W. Tu, "Visible-blind photodetector based on p-i-n junction GaN nanowire ensembles," Nanotechnology 21(31), 315201 (2010).

${ }^{14}$ H. Pettersson, I. Zubritskaya, N. Ngo Tuan, J. Wallentin, M. T. Borgstrom, K. Storm, L. Landin, P. Wickert, F. Capasso, and L. Samuelson, "Electrical and optical properties of InP nanowire ensemble p(+)-i-n(+) photodetectors," Nanotechnology 23(13), 135201 (2012).

${ }^{15}$ C. Soci, A. Zhang, B. Xiang, S. A. Dayeh, D. P. R. Aplin, J. Park, X. Y, Bao, Y. H. Lo, and D. Wang, "ZnO nanowire UV photodetectors with high internal gain,” Nano Lett. 7(4), 1003-1009 (2007).

${ }^{16}$ Y. Dan, "Optoelectronically probing the density of nanowire surface trap states to the single state limit," Appl. Phys. Lett. 106(5), 053117 (2015).

${ }^{17}$ S. Chandrasekhar, M. K. Hoppe, A. G. Dentai, C. H. Joyner, and G. J. Qua, "Demonstration of enhanced performance of an InP/InGaAs heterojunction phototransistor with a base terminal," IEEE Electron Device Lett. 12(10), 550-552 (1991).

${ }^{18}$ L. Novotny and N. van Hulst, "Antennas for light," Nature Photon. 5(2), 83-90 (2011).

${ }^{19}$ K. B. Crozier, A. Sundaramurthy, G. S. Kino, and C. F. Quate, "Optical antennas: Resonators for local field enhancement," J. Appl. Phys. 94(7), 4632-4642 (2003).

${ }^{20}$ G. K. Felic, F. Al-Dirini, F. M. Hossain, N. Thanh Cong, and E. Skafidas, "Silicon nanowire photodetector enhanced by a bow-tie antenna," Appl. Phys. A 115(2), 491-493 (2014).

${ }^{21}$ See supplementary material at http://dx.doi.org/10.1063/1.4959264 for device fabrication and simulation method.

${ }^{22}$ Y. Dan, K. Seo, K. Takei, J. H. Meza, A. Javey, and K. B. Crozier, "Dramatic reduction of surface recombination by in situ surface passivation of silicon nanowires," Nano Lett. 11(6), 2527-2532 (2011).

${ }^{23}$ S. L. Tan, X. Zhao, and Y. Dan, "High-sensitivity silicon nanowire phototransistors," Proc. SPIE 9170, 917002 (2014).

${ }^{24}$ Y. Cui, Z. Zhong, D. Wang, W. U. Wang, and C. M. Lieber, "High performance silicon nanowire field effect transistors," Nano Lett. 3(2), 149-152 (2003).

${ }^{25}$ W. I. Park, "Electrical properties of delta-doped silicon-nanowire fieldeffect transistors," J. Korean Phys. Soc. 53(4), L1759-L1763 (2008).

${ }^{26}$ D. A. Neamen, Semiconductor Physics and Devices: Basic Princples, 4 th ed. (Publishing House of Electronic Industry, 2011).

${ }^{27}$ K. E. Moselund, M. Najmzadeh, P. Dobrosz, S. H. Olsen, D. Bouvet, L. De Michielis, V. Pott, and A. M. Ionescu, "The high-mobility bended nchannel silicon nanowire transistor," IEEE Trans. Electron Devices 57(4), 866-876 (2010).

${ }^{28}$ R. A. Minamisawa, M. J. Suess, R. Spolenak, J. Faist, C. David, J. Gobrecht, K. K. Bourdelle, and H. Sigg, "Top-down fabricated silicon nanowires under tensile elastic strain up to 4.5\%," Nat. Commun. 3, 1096 (2012).

${ }^{29}$ L. Y. Cao, J. S. White, J. S. Park, J. A. Schuller, B. M. Clemens, and M. L. Brongersma, "Engineering light absorption in semiconductor nanowire devices," Nat. Mater. 8(8), 643-647 (2009).

${ }^{30}$ Hamamatsu S5627-01, S4797-01, S6931-01, S2833-01, S4011-06DS Si photodiodes datasheet.

${ }^{31}$ Hamamatsu S12023 series Si APD datasheet. 
Supporting Information

\title{
High-Performance Silicon Nanowire Bipolar Phototransistors
}

\author{
Siew Li Tan ${ }^{1, \$}$, Xingyan Zhao ${ }^{1}$, Kaixiang Chen ${ }^{1}$, Kenneth B. Crozier ${ }^{2}$, Yaping Dan ${ }^{*}, 1$ \\ ${ }^{1}$ University of Michigan-Shanghai Jiao Tong University Joint Institute, Shanghai Jiao Tong \\ University, Shanghai, 200240, P. R. China \\ ${ }^{2}$ School of Engineering and Applied Sciences, Harvard University, Cambridge, MA 02138, USA \\ ${ }^{\S}$ Currently with the CEA Grenoble, 17 rue des Martyrs, 38054 Grenoble, France. \\ *E-mail: yaping.dan@sjtu.edu.cn
}

\section{S1. Device fabrication method}

SiNW phototransistor devices were fabricated on a silicon-on-insulator (SOI) wafer using a top-down approach. The SOI wafer consists of a (100)-oriented, $200 \pm 10 \mathrm{~nm}$ thick, boron-doped silicon ( $\mathrm{Si}$ ) device layer with a resistivity of $10-20 \Omega \mathrm{cm}$. The thickness of the oxide layer below the Si device layer is $375 \pm 10 \mathrm{~nm}$. Prior to device processing, the wafer was cleaned in piranha $\left(\mathrm{H}_{2} \mathrm{SO}_{4}: \mathrm{H}_{2} \mathrm{O}_{2}\right)$ and buffered oxide etch $\left(\mathrm{NH}_{4} \mathrm{~F}: \mathrm{HF}\right)$ solutions. Arrays of single nanowires were patterned on the wafer by electron beam lithography (EBL). Following EBL, a thin layer of aluminum (Al) film was thermally evaporated on the wafer and lifted off to form a metal etch mask. The nanowires were formed by etching the Al-masked Si device layer down to the top of 
the oxide layer, using a $\mathrm{SF}_{6} / \mathrm{C}_{4} \mathrm{~F}_{8}$ inductively coupled plasma reactive ion etching (ICP-RIE) process. Subsequently, the $\mathrm{Al}$ mask was removed using standard $\mathrm{Al}$ etchant.

Figure S1.1 illustrates the pnp phototransistor device structure, which comprises a $200 \mathrm{~nm}$ wide and $3 \mu \mathrm{m}$-long nanowire connected at each end to a large contact pad. The pnp device consists of $1-\mu \mathrm{m}$-long $p^{+}$-doped emitter, $n$-doped base with length ranging from 100 to $500 \mathrm{~nm}$, 1 - $\mu \mathrm{m}$-long $p^{-}$-doped collector, and 1- $\mu \mathrm{m}$-long $p^{+}$-doped sub-collector. A $p^{+}$-doped contact pad is connected to the end of the emitter and sub-collector regions, respectively. Additionally, $p^{+}-n$ SiNW diodes with dimensions similar to the pnp phototransistors were also fabricated on the same wafer. The $p^{+}-n$ SiNW diode structure is shown in Figure S1.2. Since the $p^{+}-n$ diode functions as a unity-gain photodiode at low bias voltages, it provides the baseline from which the photocurrent gain of the pnp SiNW phototransistors can be defined.

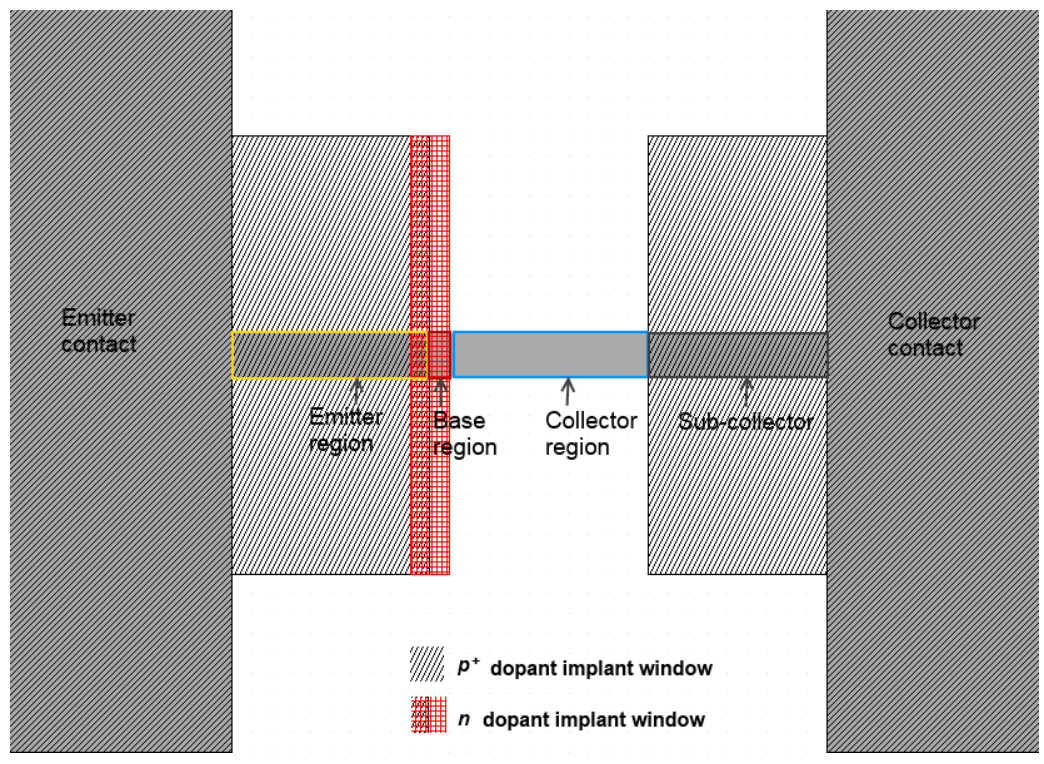

Figure S1.1. Schematic of the pnp SiNW phototransistor device structure. The diagonal lines and grid lines represent the boron ( $p^{+}$-type) and phosphorus ( $n$-type) ion implantation windows, respectively. 


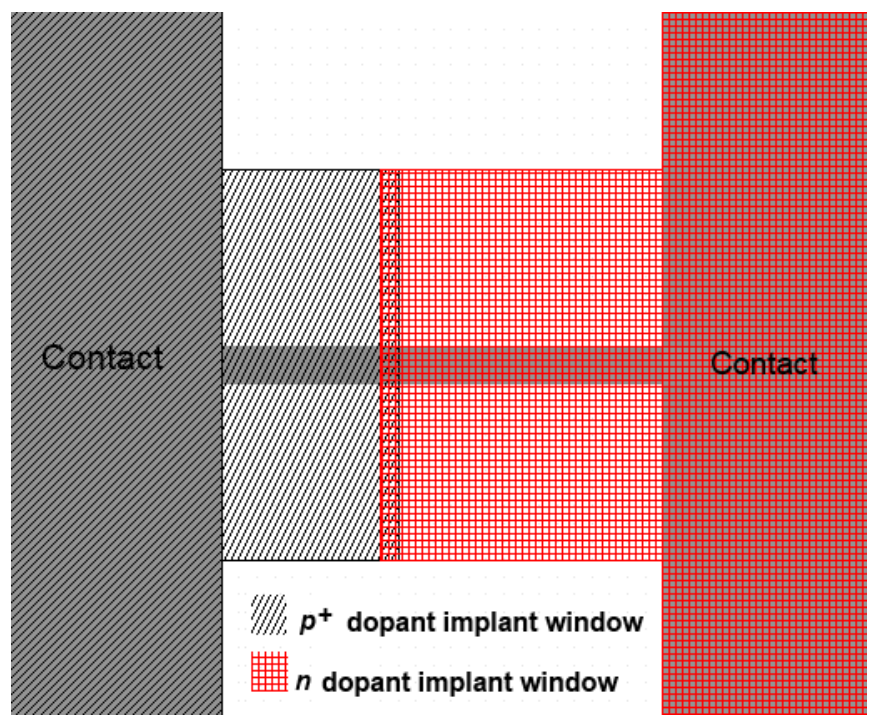

Figure S1.2. Schematic of the $p^{+}-n$ SiNW diode structure which serves as a unity-gain device in this work. The diagonal lines and grid lines represent the boron $\left(p^{+}\right.$-type) and phosphorus ( $n$-type) ion implantation windows, respectively.

The doping of the $p^{+}$and $n$-type active regions of the SiNW devices fabricated in this work was defined, respectively, by boron and phosphorus ion implantation. The energy and dose values are $20 \mathrm{keV}, 8 \times 10^{14} \mathrm{~B}^{+} \mathrm{cm}^{-2}$ for boron, and $80 \mathrm{keV}, 1 \times 10^{13} \mathrm{P}^{+} \mathrm{cm}^{-2}$ for phosphorus. Each of the two ion implantation steps was performed separately, following cleaning and EBL patterning of implantation windows on the wafer with polymethylmethacrylate (PMMA) resist. The PMMA resist has sufficient density and thickness to act as a robust mask. To correct for possible EBL alignment error (estimated to be $<100 \mathrm{~nm}$ ), the base ion implantation window was extended by $100 \mathrm{~nm}$ into the emitter region. The overlapped region has negligible effect on the emitter-base junction profile as the emitter region is significantly more highly doped than the base region. 


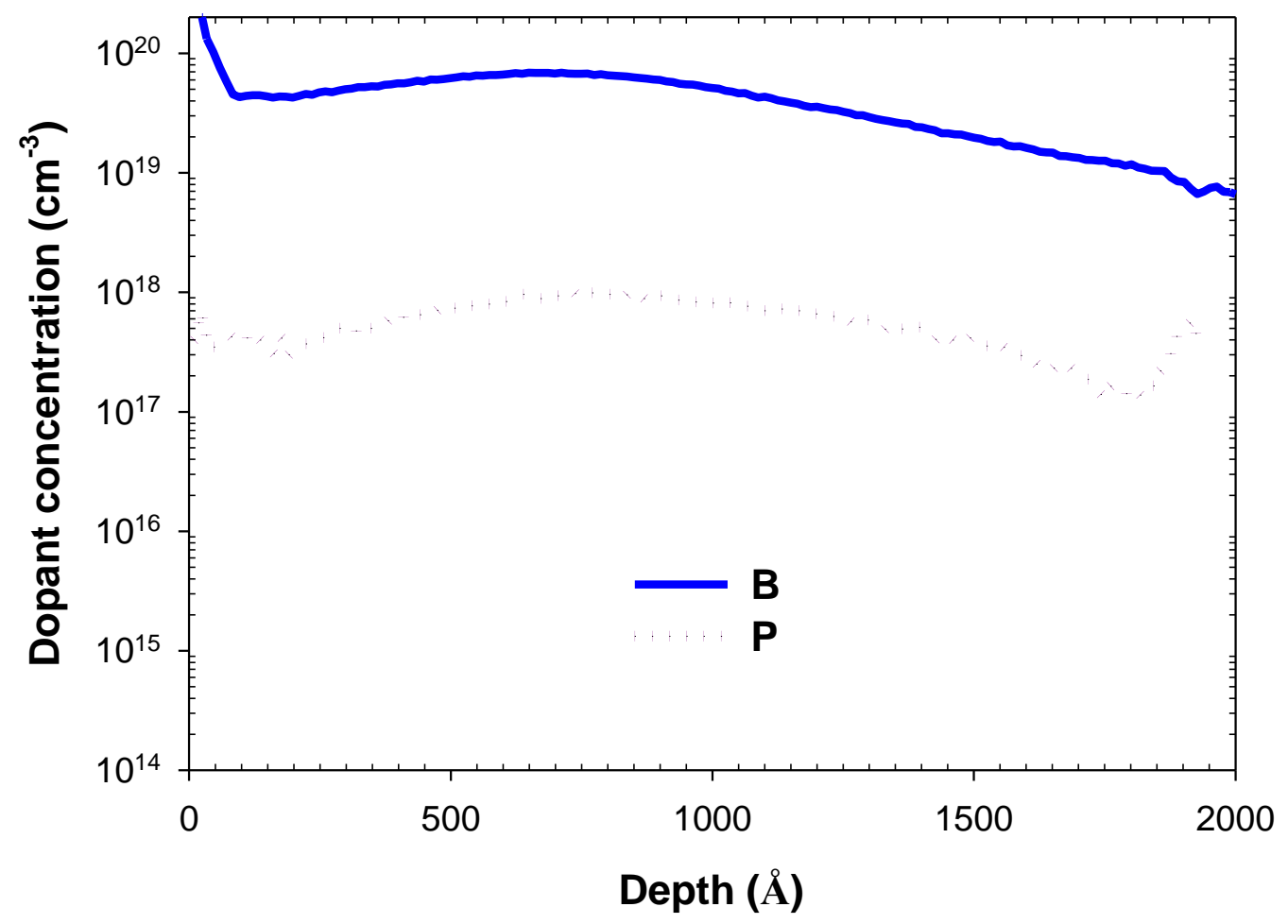

Figure S1.3. Boron (solid line) and phosphorus (dotted line) ion-implanted doping profiles measured by SIMS following RTA annealing of as-implanted sample. The depth value of 0 represents the top surface.

Following ion implantation, both the boron and phosphorus dopants were activated simultaneously by rapid thermal annealing (RTA) at $1000{ }^{\circ} \mathrm{C}$ in an $\mathrm{N}_{2}$ ambient. Figure $\mathrm{S} 1.3$ shows the secondary ion mass spectroscopy (SIMS) profiles of the annealed boron and phosphorus ion-implanted regions. The average doping concentration in the $p^{+}$(emitter, subcollector and contact) and $n$-type (base) regions is approximately $5 \times 10^{19} \mathrm{~cm}^{-3}$ and $5 \times 10^{17} \mathrm{~cm}^{-3}$, respectively. Finally, Ti/Au ohmic contacts were thermally evaporated on the emitter and subcollector contact pad windows of the devices. 


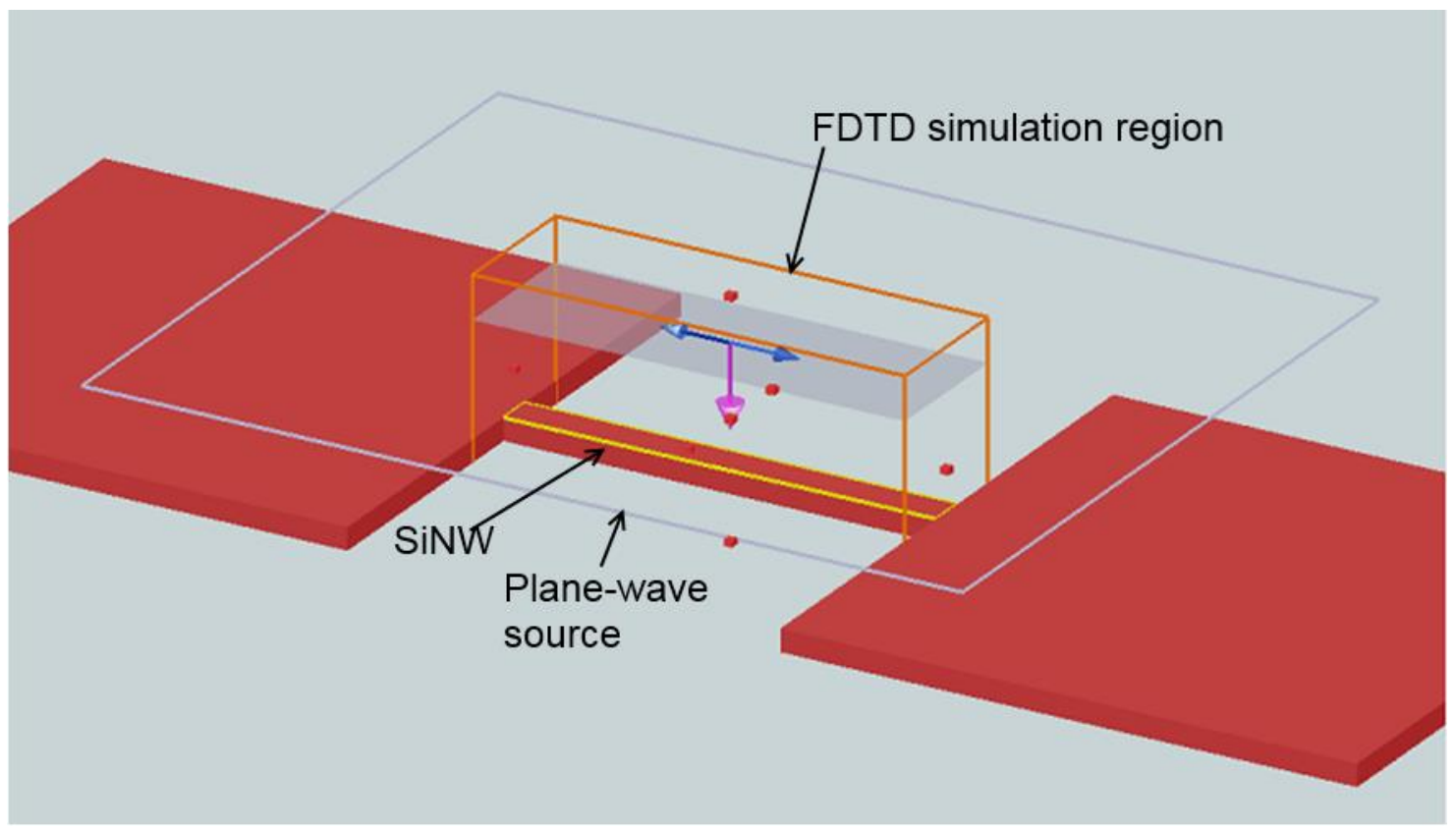

Figure S2.1. Schematic of FDTD simulation structure.

\section{S2. Simulation method}

In order to compare the experimental device performance with theoretical prediction, the electro-optical characteristics of the pnp SiNW phototransistor were simulated using the Lumerical DEVICE and FDTD software packages. A schematic of the finite-difference timedomain (FDTD) simulation structure is shown in Figure S2.1, which consists of a SiNW topilluminated by a plane-wave source. Perfectly-matched-layer (PML) boundary conditions are used in the $z$ direction (normal to the top absorbing surface of the SiNW), while periodic boundary conditions are defined in the $x$ (NW length) and $y$ (NW width) directions. The FDTD simulation produces the photo-generation profile which was incorporated into the electrical simulation performed with Lumerical DEVICE.

In the simulated $p n p \mathrm{SiNW}$ device structure, the doping concentration of the $p^{-}$-doped collector region is equivalent to $1 \times 10^{15} \mathrm{~cm}^{-3}$, as estimated from the resistivity value of the silicon device 
layer of the SOI wafer, and assumed to be uniform in all directions. The doping concentrations of the $p^{+}$-doped emitter, sub-collector and contact regions and the $n$-doped base region are assumed to be uniform in the $x$ and $y$ directions and varying in the $z$ direction according to the doping profiles given in Figure S1.3. Bulk (Shockley-Read-Hall) and surface recombination mechanisms are included in the electrical simulation. In addition, $p^{+}-i-n^{+}$and $p^{+}-n$ SiNW diode structures with, respectively, $i$-region $\left(p^{-}\right.$-doped at $\left.1 \times 10^{15} \mathrm{~cm}^{-3}\right)$ and $n$-region $\left(n\right.$-doped at $5 \times 10^{17}$ $\mathrm{cm}^{-3}$ ) length comparable to the collector length of the pnp SiNW phototransistor, were also simulated for comparison with the pnp device.

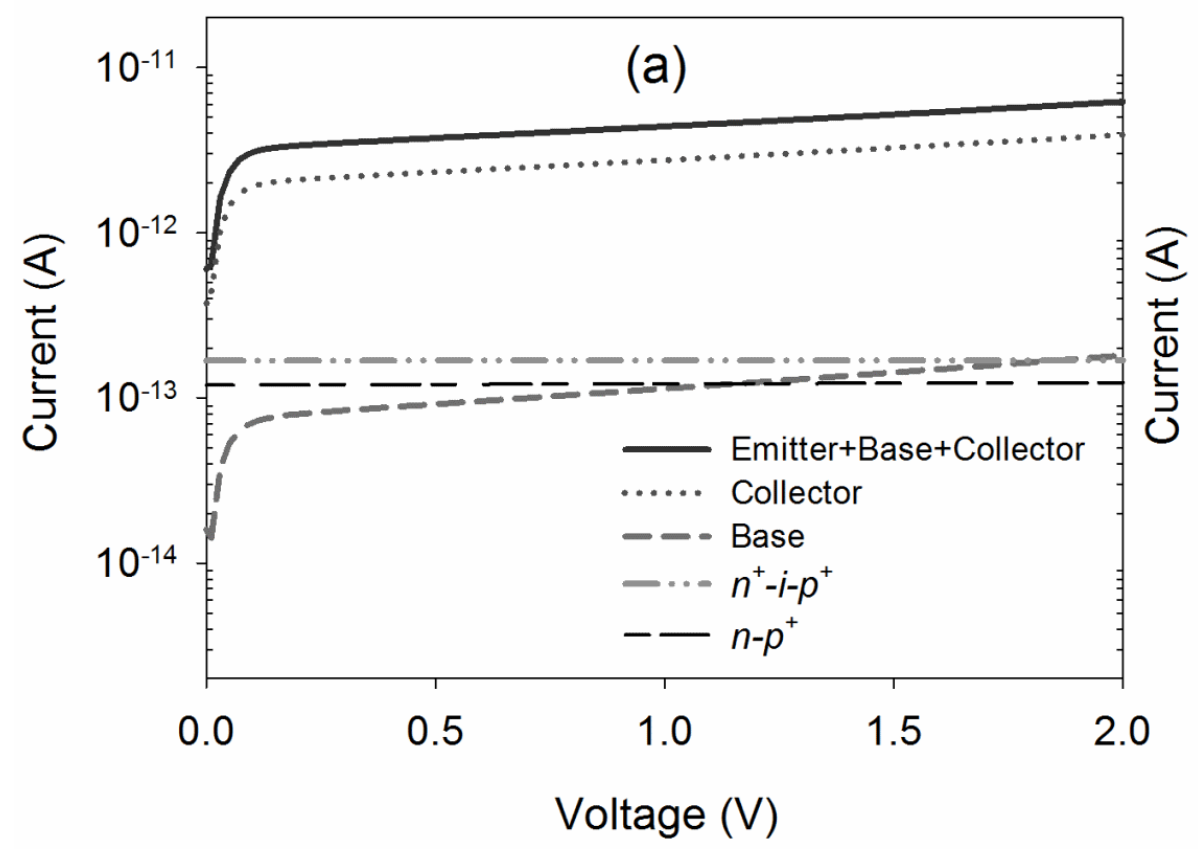

Figure S2.2. Simulated photocurrents in a SiNW phototransistor (100-nm base, $1-\mu \mathrm{m}$ collector) with only the base, collector, or the whole SiNW active region under illumination. Also shown are simulated photocurrents of $n^{+}-i-p^{+}$diode with $1 \mu \mathrm{m} i$-region and $n-p^{+}$diode with $1.5 \mu \mathrm{m} n$ region, for comparison. The incident light is $532 \mathrm{~nm}$ at $\sim 160 \mathrm{~W} \mathrm{~cm}^{-2}$. 